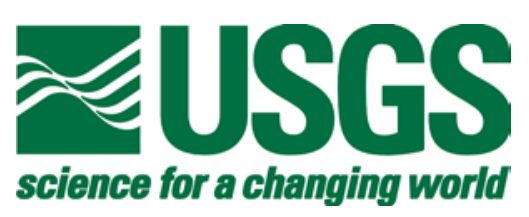

\title{
Geosoft eXecutables (GX's) developed by the U.S. Geological Survey, version 1.0, with a viewgraph tutorial on GX development
}

By Jeffrey D. Phillips ${ }^{1}$, Joseph S. Duval ${ }^{2}$, and R.W. Saltus ${ }^{1}$

${ }^{1}$ Denver, CO 80225

${ }^{2}$ Reston, VA 20192

This report is preliminary and has not been reviewed for conformity with U.S. Geological Survey editorial standards. Any use of trade, product, or firm names is for descriptive purposes only and does not imply endorsement by the U.S. Government.

Mention of trade names or commercial products does not constitute endorsement or recommendation for use by the U.S. Government.

Although these programs have been used by the U.S. Geological Survey, no warranty, expressed or implied, is made by the USGS as to the accuracy and functioning of the programs and related program material nor shall the fact of distribution constitute any such warranty, and no responsibility is assumed by the USGS in connection therewith.

\section{Open-File Report 03-010}

U.S. Department of the Interior

U.S. Geological Survey 


\section{Introduction}

Geosoft executables (GX's) are custom software modules for use with the Geosoft OASIS/Montaj ${ }^{\mathrm{TM}}$ geophysical data processing system, which runs under the Microsoft Windows ${ }^{\mathrm{TM}}$ operating system. The U.S. Geological Survey (USGS) uses OASIS/Montaj, primarily for processing and display of airborne geophysical data. The ability to add custom software modules to the OASIS/Montaj system is a feature advantageous to the USGS, due to the large number of geophysical algorithms developed by the USGS during the past half century.

This report describes the initial set of GX's developed by the USGS or specifically for the USGS by contractors. They perform fairly basic operations that were missing or perceived to be inadequate in the built-in GX's that came with OASIS/Montaj version 4.2. Some of these GX's or GX's with similar functionality may be incorporated into later versions of OASIS/Montaj.

\section{Download and Installation}

The executable GX files, which will only work from within Geosoft OASIS/Montaj, and the source code files used to develop them can be downloaded using a web browser from ftp://ftpext.usgs.gov/pub/cr/co/denver/musette. The files are in the /pub/gx directory.

Each compiled GX consists of an executable file with the suffix .gx. In addition there is a global file containing error messages called usgs.err and a menu file called usgs.omn. Some GXs require a dynamically linked library with the suffix .dll. In the current release of OASIS/Montaj (version 5.1.5), compiled GXs are installed in the gx subdirectory of the IOASISmontaj directory; error files are installed in the ger subdirectory; menu files are installed in the omn subdirectory, and dynamic-link libraries are installed in the bin subdirectory.

\section{The USGS Menu File and Short GX Descriptions}

The GX's described here can be accessed from a USGS menu that can be added to the menu bar in OASIS/Montaj. The menu structure is defined in a text file called usgs.omn:

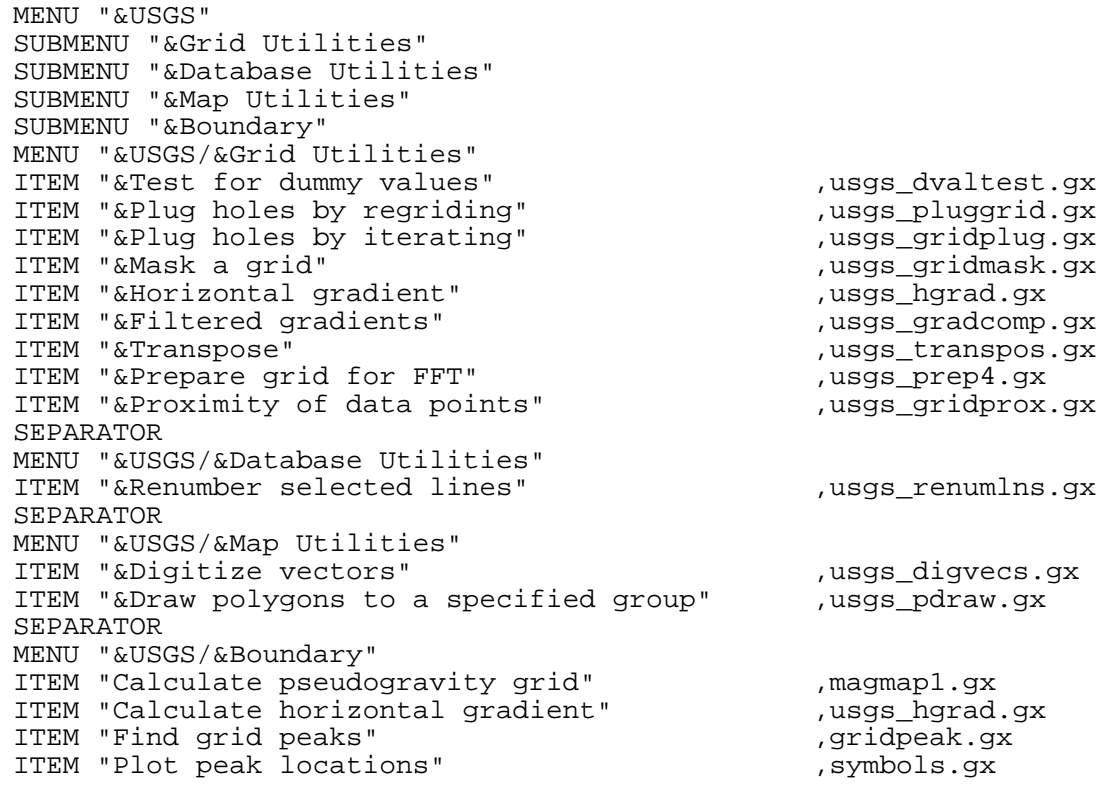

This file can be easily modified to add additional categories and custom GX's as they are developed. The magmap1.gx, gridpeak.gx, and symbols.gx referenced in the menu file are part of the OASIS/Montaj package.

The following GX's are described in this report: 
usgs_digvecs.gX - digitize vectors from a map and place them in an ASCII XYZ file.

usgs_dvaltest.gx - test for dummy values in a grid.

usgs_gradcomp.gx - compute filtered horizontal derivative grids and the horizontal gradient magnitude grid using the gradient-component method of Thurston and Brown (1994).

usgs_gridmask.gx - restore holes to a grid from a masking grid.

usgs_gridplug.gx - plug holes in a grid by using minimum curvature iterations.

usgs_gridprox.gx - create a grid containing distance to the nearest data point.

usgs_hgrad.gx - calculate the magnitude of the horizontal gradient of a grid.

usgs_pdraw.gx - draw, redraw, or append polygons from a .ply file to a specified map group.

usgs_pluggrid.gx - plug holes in a grid by regriding.

usgs_prep4.gx - prepare a grid for Fourier transform by plugging holes (if any), and extending the rows and columns.

usgs_renumlns.gx - renumber selected lines in a database.

usgs_transpos.gx - transpose a grid. 


\title{
Help Files for the Individual GX's
}

\section{USGS_DIGVECS.HLP}

\author{
USGS_DIGVECS GX \\ Digitize vectors from a map and place them in an ASCII XYZ file.
}

INTERACTIVE PARAMETERS

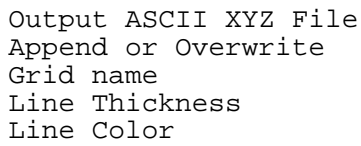

BATCH PARAMETERS

$\begin{array}{ll}\text { USGS_DIGVECS.XYZ } & \text { output ASCII XYZ file name } \\ \text { USGS_DIGVECS.APPEND } & \text { overwrite file }=0 ; \text { append to output = } 1 \\ \text { USGS_DIGVECS.GRID } & \text { grid name } \\ \text { USGS_DIGVECS.LINETHICK } & \text { line thickness (mm) } \\ \text { USGS_DIGVECS.LINECOLOR } & \text { line color }\end{array}$

APPLICATION NOTES

If the output file does not exist, you must specify overwrite mode. If the file does exist and you specify overwrite mode, you will be asked to confirm the overwrite.

If no grid is specified, only LINE, $X, Y$ coordinates will be placed in the output file. If a grid is specified, LINE,X, Y and Z values will be placed in the output file. A header record in the output file identifies the channel names. To use this header record when importing the XYz file into a database, blank out the 'Import template' field.

A map group corresponding to the output file name prefix will be used to hold the vectors drawn on the map.

Multi-point vectors are digitized using the left mouse button. The right mouse button is used to terminate a vector (using the "Done" menu item) and start a new vector. Digitizing is terminated by using the right mouse button and selecting "Done" twice in a row. The "Cancel" menu item is equivalent to the "Done" menu item, it does not remove the digitized vector from the file or the map. The "Exit Menu" menu item will cancel the menu and return to digitizing the current vector.

Because the map cannot be redrawn inside a GX, the digitized vectors will not be drawn until the GX is exited. For the same reason, the remaining right mouse menu items won't work properly.

Written by Jeff Phillips (jeff@usgs.gov) 2/9/99, modified 3/21/02. 


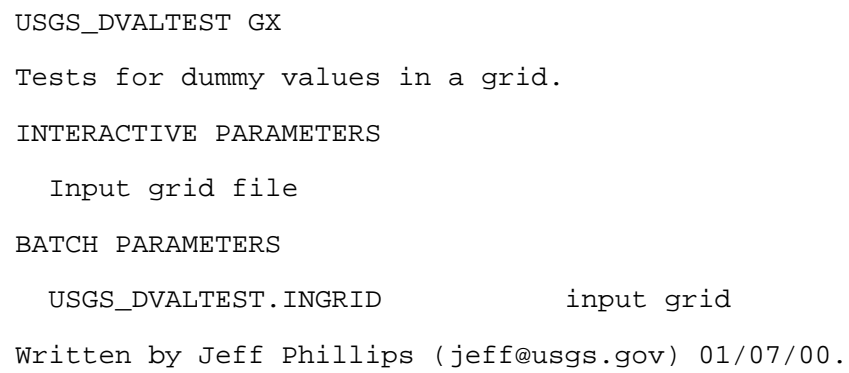

\section{USGS_GRADCOMP .HLP}

USGS_GRADCOMP GX

Computes filtered horizontal derivative and horizontal gradient

magnitude (HGM) grids using the gradient-component method of

Thurston and Brown (1994).

INTERACTIVE PARAMETERS

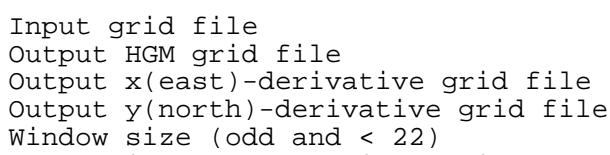

\section{BATCH PARAMETERS}

$\begin{array}{ll}\text { USGS_GRADCOMP.GRID } & \text { input grid } \\ \text { USGS_GRADCOMP.HGRID } & \text { output HGM grid } \\ \text { USGS_GRADCOMP.XGRID } & \text { output x-derivative grid } \\ \text { USGS_GRADCOMP.YGRID } & \text { output y-derivative grid } \\ \text { USGS_GRADCOMP.WINDOW } & \text { window size } \\ \text { USGS_GRADCOMP.POLY } & \text { polynomial order }\end{array}$

APPLICATION NOTES

Computes filtered horizontal derivative and horizontal gradient magnitude grids using the gradient-component method of Thurston and Brown (1994). The user must specify a window size w and a polynomial order $\mathrm{n}<\mathrm{w}$. The standard first-difference operator corresponds to $\mathrm{w}=3, \mathrm{n}=2$. For this and similar operators having $\mathrm{n}=\mathrm{w}-1$, there is no attenuation of short wavelengths. For operators having $\mathrm{n}<\mathrm{w}-1$, short wavelength features will be attenuated.

Source code modified from Thurston and Brown (1994) by Jeff Phillips. The maximum number of columns in the grid is limited to 8000 .

Reference :

Thurston, J.B., and Brown, R.J., 1994, Automated source-edge location with a new variable pass-band horizontal-gradient operator: Geophysics, v.59, no.4, p.546-554.

Written by Jeff Phillips (jeff@usgs.gov) 10/13/99.

\section{USGS_GRIDMASK.HLP}

USGS_GRIDMASK GX

Masks a grid file by inserting dummy values from a second grid file (normal masking) or by inserting the non-dummy values from the second grid file as dummy values in the masked grid file (inverse masking).

INTERACTIVE PARAMETERS 
Grid file to be masked

Grid file containing the masking values

Output masked grid file

Normal or inverse masking

BATCH PARAMETERS

USGS_GRIDMASK.INGRID1

USGS_GRIDMASK. INGRID2

USGS_GRIDMASK.OUTGRID

input grid file to be masked

input masking grid file

USGS_GRIDMASK.MTYPE

output masked grid file

type of masking (Normal or Inverse)

APPLICATION NOTES

The maximum column dimension is 16384 for all grids.

Written by Jeff Phillips (jeff@usgs.gov) 01/18/00.

\section{USGS_GRIDPLUG.HLP}

USGS_GRIDPLUG GX

Plugs dummy values in a grid file by using polynomial or local median initialization followed by minimum curvature iterations.

INTERACTIVE PARAMETERS

Grid file to be plugged

Output plugged grid file

Polynomial order ( 0 to 3 , or -1 for local median replacement)

Number of minimum curvature iterations

BATCH PARAMETERS

USGS_GRIDPLUG.INGRID

USGS_GRIDPLUG.OUTGRID

USGS_GRIDPLUG.IORDER

input grid file to be plugged

output plugged grid file

polynomial order for initialization

( 0 to 3 or -1 for local median)

USGS_GRIDPLUG.NITER

number of minimum curvature iterations

(e.g., 100)

APPLICATION NOTES

The maximum column dimension of the grids is 10,000.

Written by Jeff Phillips (jeff@usgs.gov) 12/16/2002 . 


\section{USGS_GRIDPROX.HLP}

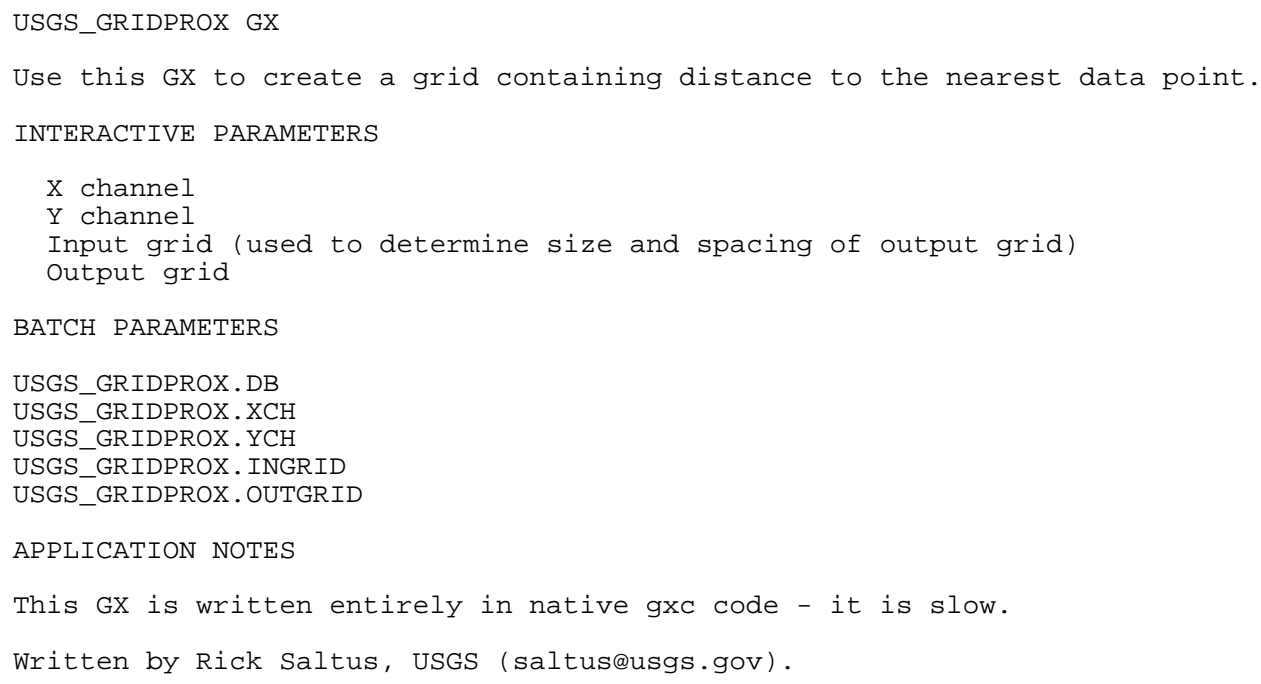

\section{USGS_HGRAD.HLP}

USGS_HGRAD.GX

This GX calculates the magnitude of the horizontal gradient of a grid.

INTERACTIVE PARAMETERS

Input grid

Output horizontal gradient magnitude grid

BATCH PARAMETERS

USGS_HGRAD.IN = Input grid

USGS_HGRAD.OUT = Output horizontal gradient magnitude grid

APPLICATION NOTES

The HORIZONTAL GRADIENT MAGNITUDE is the square root of the sum of the squares of the derivatives in the $x$, and $y$ directions:

hgrad $=\operatorname{sqrt}(d x * d x+d y * d y)$

Written by Northwest Geophysical Associates for the USGS. 


\section{USGS PDRAW. HLP}

The USGS_PDRAW GX draws the polygons from a polygon file on the map.

Draw polygons from a polygon file dialog box options

Polygon file name
Map view
line thickness (mm)
Line colour
Fill colour
Group Name
Group Action

The name of the polygon file. Script Parameter: USGS_PDRAW.FILE

Map view in which to draw, select from the list.

Script Parameter: USGS_PDRAW.VIEW

The polygon outline line thickness in $\mathrm{mm}$. Script Parameter: USGS_PDRAW.LINETHICK

Enter a line colour of form RxxxGxxxBxxx. Script Parameter: USGS_PDRAW.LINECOLO

Enter a fill colour of form RxxxGxxxBxxx. Script Parameter: USGS PDRAW.FILLCOLO

Enter the group name Script Parameter: USGS PDRAW.GROUPNAME

Enter a zero (0) to replace the group (i.e. Erase and replot) and a one (1) to append polygons to the group. Script Parameter: USGS_PDRAW.GROUPACTN

Application Notes

Polygons are ASCII files with default extension .ply. A polygon file contains a list of X, Y coordinates that define one or more polygons. The file may contain any number of polygons, and each polygon may have any number of vertices. The first and last points in each polygon are assumed to connect. If the file will contain more than one polygon, each polygon must start with a line 'poly \#' ('p' or 'P' in column 1). Comment lines are indicated by a '/' in column 1. Please note that the polygon coordinates are assumed to be in the same coordinate system as that of the map view on which the polygons will be drawn.

Following is an example of a single polygon file:

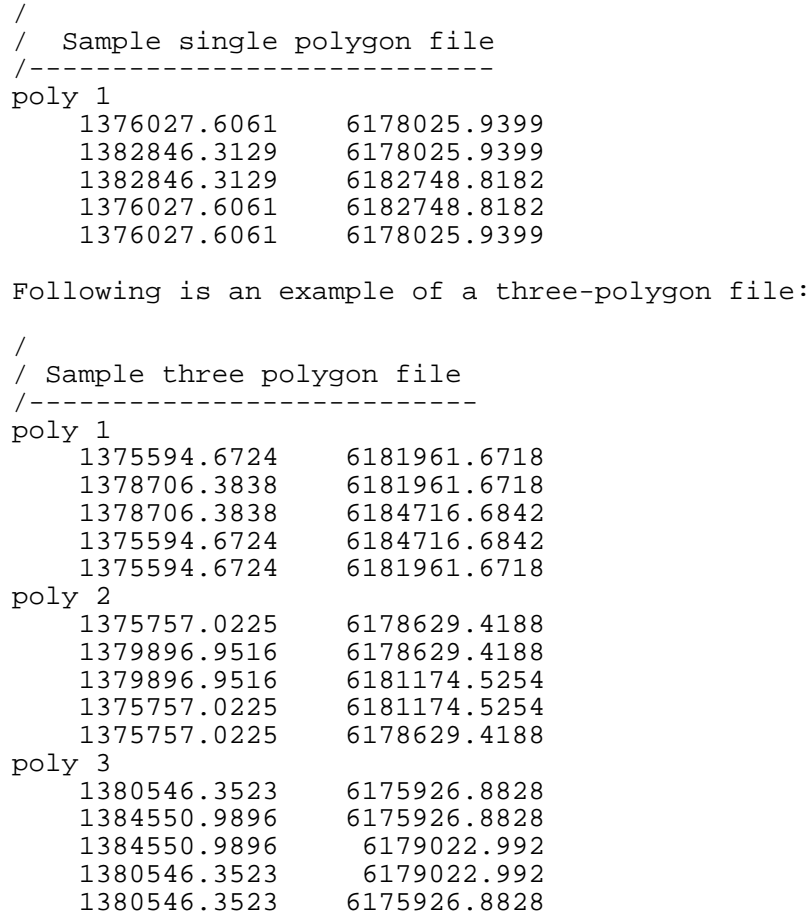

TOPIC MODIFIED BY JOE DUVAL, USGS (03/04/2002) 


\section{USGS_PLUGGRID.HLP}

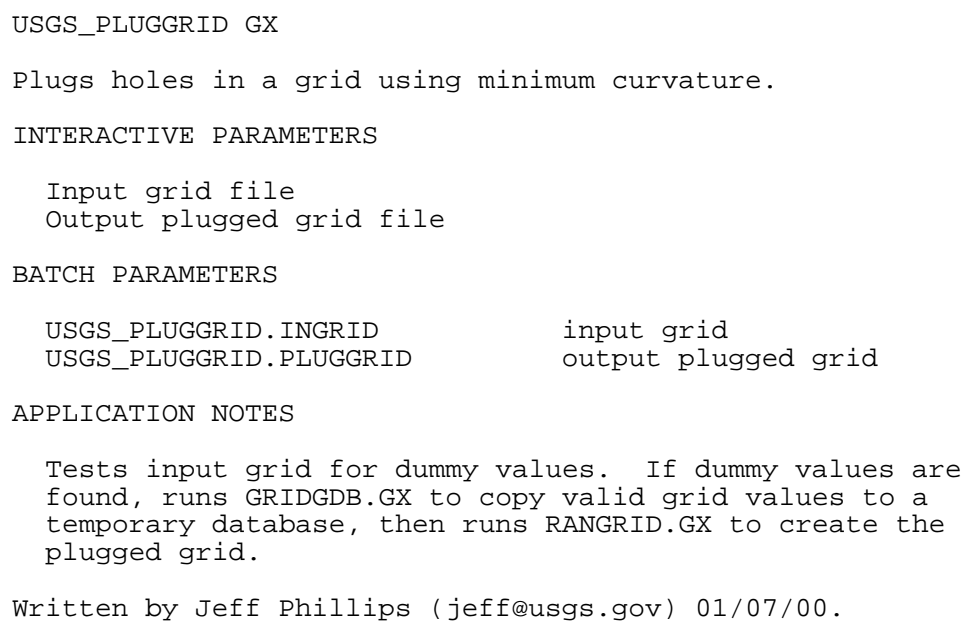

\section{USGS_PREP 4 .HLP}

USGS_PREP 4 GX

Prepares a grid for Fourier transform by plugging holes, and extending the rows and columns.

INTERACTIVE PARAMETERS

Input grid file

Output plugged and extended grid file

Percent expansion

BATCH PARAMETERS

USGS_PREP 4.INGRID

USGS_PREP 4.PREPGRID

input grid

USGS_PREP 4.PCT

output plugged and extended grid percent expansion

APPLICATION NOTES

Maximum dimensions of the extended grid are $16384 \times 16384$. The input grid should be smaller than this.

Initial processing tests the input grid for dummy values; if any exist, the valid grid values are copied into a temporary database called

Prep4.gdb and regridded using minimum curvature into a new grid called

Prep4.plg.

The input grid (or Prep4.plg) is then processed through the

following 8 steps:

1. Get the min/max of the grid and the extended dimensions.

2. Extend the grid to the right using prediction filtering.

3. Transpose the extended grid.

4. Extend the transposed grid to the right.

5. Smooth across the first extension.

6. Transpose back.

7. Smooth across the second extension.

8. Center the data in the grid.

Written by Jeff Phillips (jeffeusgs.gov) 01/18/00.

\section{USGS_RENUMLNS.HLP}

Use the USGS_RENUMLNS GX to renumber all selected lines. This is useful for changing line numbers prior to merging databases. 
Renumber all selected lines dialog box options

Starting Line Number

Enter the starting line number. Script Parameter: USGS_RENMULNS.STRTLN

Line number Increment

Enter the increment to be used for numbering sequential lines.

Script Parameter: USGS_RENUMLNS.INCRLN

Application Notes

If any parameters are blank, the default parameter value is set to 1 .

The process will stop if the new name of a selected line already exists.

All lines processed up to that point will be changed.

TOPIC WRITTEN BY JOE DUVAL, USGS $(11 / 21 / 2000)$

\section{USGS TRANSPOS. HLP}

USGS_TRANSPOS GX

Transpose a grid.

INTERACTIVE PARAMETERS

Input grid file

Output grid file

BATCH PARAMETERS

USGS_TRANSPOS.INGRID input grid

. OUTGRID output grid

APPLICATION NOTES

Written by Jeff Phillips (jeff@usgs.gov) 1/11/00. 


\section{Source Code Files and Tutorials}

Each GX is built from a number of source code files. These include, at a minimum, an ASCII help file with the suffix .hlp, a GX source code file with the suffix .gxc, and a resource file with the suffix .grc. Some GXs use a dynamic link library compiled from Fortran code. In addition to the three source code files described above, these GXs require the Fortran source code file with a suffix of .f, which is converted to C code using Geosoft's version of f2c.exe; a C code wrapper function for the Fortran subroutine calls, usually called $\mathbf{g x x}_{-}{ }^{*}$.c ; the generic wrapper functions wfuncs.c; the $\mathrm{C}$ header files f2c.h and wrappers.h, and a .gxh prototype file. The interrelationships of these files are described in the following tutorial on GX development, which is suitable for printing in viewgraph format. 


\section{SIMPLE GX DEVELOPMENT}

Files you supply

Help (.HLP)

Resource (.GRC)

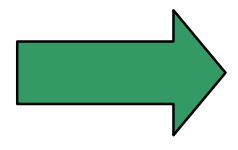

Source $(. G \times C)$

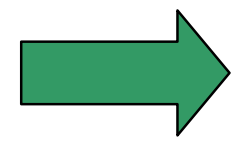

GX Compiler

(GXC.EXE)

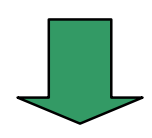

Geosoft Executable (.GX)

Menu Definition (.OMN) 


\section{STEPS IN DEVELOPMENT}

1. Decide exactly what the GX will do. Choose a name for the GX. Determine the input required from the user.

2. Create a new $G X$ project directory and copy an existing, somewhat similar $G X$ source code $(. G X C)$ file, resource (.GRC) file, and help (.HLP) file to be used as prototypes. Rename the files to the name of the new $G X$.

3. Edit the resource (.GRC) file and the help (.HLP) file to reflect your design. Run the GRC resource compiler, which is in the $\backslash$ OASISmontaj \bin directory on the PC. Correct syntax errors and warnings.

4. Edit the source code $(G X C)$ file to perform the required function. Remember to correct the GX name in the description and the names of the included resource files. Note: library routines are poorly documented; see the header (.GXH) files in the INCLUDE directory on the CD-ROM and the source code examples under the SRC directory. Compile using the GXC source compiler, also in the \OASISmontaj\bin directory on the PC. Correct errors.

5. Move the compiled GX to the \OASISmontajlgx directory. Start Oasis and test the GX. Correct any errors you see at run time.

6. Include the $G X$ in a new or existing menu definition (.OMN) file in the IOASISmontaj lomn directory for easier access from the Oasis menu bar. 


\section{WHAT'S IN THE USER-SUPPLIED FILES?}

Help File (mygx.HLP) - text only; should describe variables and the function of the entire GX.

Resource File (mygx.GRC) - Defines the user interface. Components include:

// - comment lines

FORM - A dialog box with one or more of:

EDIT - text entry fields

FEDIT - filename entry fields

LEDIT - drop-down lists

EBUT - pushbuttons

HBUT - help pushbuttons

LIST - drop-down list definition for each LEDIT with two or more:

ITEM - list items

HELP - pointer to the help file

Source File (mygx.GXC) - pseudo-C code with the following sections:

Description

NAME = "MY GX"

VERSION = "v1.00a US Geological Survey"

DESCRIPTION = "A multi-line text description"

Resources

RESOUCE = "mygx.gr"

\#include "mygx.grh"

Includes

\#include <all32.grh>

Variable definitions

strings, reals, ints, DGW (dialog objects), etc. 
Code

\{

// Interactive Parameter Block specification

if (iInteractive_SYS()) \{ // Are we running interactively?

Diag = Create_DGW("MYGX"); // Create the dialog SetInfoSYS_DGW(Diag,...);// Set default parameters

$i=$ iRunDialog_DGW(Diag); // Run dialog GetInfoSYS_DGW(Diag,...);// Load new parameters

\}

// Read Parameter Block

GetString_SYS("MYGX",...); // Get strings iVar = iGetInt_SYS("MYGX",...); // Get integers rVar = rGetReal_SYS("MYGX",...); // Get reals

// Initialize objects

// Process the data

// Cleanup and exit

\}

Menu Definition File (USGS.OMN)

I

I USGS

/----------

MENU "\&USGS"

SUBMENU "\&Digitizing"

MENU "\&USGS/\&Digitizing"

ITEM "\&digitize vectors"

mygx.gx

SEPARATOR 


\section{CONVERTING THE EXAMPLE FORTRAN CODE TO A GX USING GEOSOFT'S F2C AND LIBRARIES}

REQUIRED: $\quad$ Microsoft Visual $C++$ version 6.0

1. Start Visual $\mathrm{C}_{++}$and create a new Project/Workspace using

File / New / Win32 Dynamic-Link Library.

Give the new project a name that corresponds to the DLL name in the .gxh file ("example" in this case) and a location. Note that OASIS/Montaj doesn't like DLL names with prefixes longer than 11 characters.

Create an empty DLL project.

The result will be a new directory (example) containing files example.dsp, example.dsw, and example.ncb

2. Close or iconify Visual $\mathrm{C}_{++}$.

3. Copy the Fortran source code (example.f) to the new directory.

(If this were your code, you would need to edit it to:

a. replace all user I/O with variables passed through subroutine calls.

b. replace all file I/O with calls to wrapper functions.)

4. Run Geosoft's f2c.exe as:

$\mathrm{f} 2 \mathrm{c}-\mathrm{A}$ example.f

This will create example.c in your directory.

5. Copy wfuncs.c, gxx_example.c, wrappers.h, and f2c.h to your directory. (If this were your code, you would need to create or modify the first three files.) 
6. Restart Visual $C_{++}$and build the project using:

Project / Add to Project / Files - add all files.

Project / Settings / Link - add geogx.lib to the end of the object/library modules list.

Tools / Options / Directories*

include files - add C: \OASISmontaj $\backslash G \times$ Dev $\backslash$ Fortran $\backslash f 2 c$

$C: \backslash O A S I S m o n t a j \backslash G \times D e v \backslash C \backslash$ include

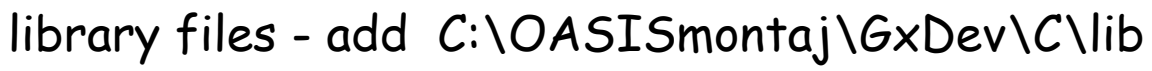

Build / Build example.dll

7. Close or iconify Visual $C_{++}$

8. Copy the new dynamic -link library (example.dll) from the Debug subdirectory to the working directory.

9. Copy example.rtf, example.grc, example.gxc, and example.gxh to your working directory.

10. Run grc example.

11. Setup your environment:

set include $=C: \backslash O A S I S m o n t a j \backslash G \times D e v \backslash$ include

12. Run gxc example.

13. Copy example.gx to the C: \OASISmontaj $\backslash$ gx directory, and example.dll to the C:\OASISmontaj\bin directory.

14. Test it from within Oasis.

* You only need to do this once. 


\section{CONVERTING YOUR FORTRAN CODE TO A GX USING GEOSOFT'S F2C AND LIBRARIES}

1. Follow the example (example.f), and use the supplied wrapper functions (example lwfuncs.c and wrappers.h).

2. You need the following files to create the DLL:

yourcode.f - edited to remove or replace I/O with parameters passed through subroutine calls or with calls to wrapper functions.

yourcode.c - the result of $f 2 c-A$ yourcode.f

f2c.h - copy it from the example directory.

$g x x$ yourcode.c - wrapper function for IUSGS_yourcode.

wfuncs.c - the _WF wrapper functions.

wrappers.h - wrapper include files.

3. You need the following files to create the gx:

yourcode.dll - produced by Visual $C_{++}$6.0.

yourcode.grc - resource source.

yourcode.gxc - gx source.

yourcode.gxh - gx include file - defines the DLL name.

yourcode.err or yourcode.ger - optional error messages.

4. Once the $g x$ is compiled, the following files need to be copied to the C:\OASISmontaj Igx \bin and Iger subdirectories respectively:

yourcode.gx - the compiled gx

yourcode.dll - the dynamic link library

yourcode.err or yourcode.ger - optional error messages 


\section{WHAT TO DO IF IT DOESN'T WORK}

1. Check the Fortran code:

a. Make sure you have the correct number of file I/O calls to wrapper functions.

b. Make sure the variables in the wrapper function calls are passed in the correct order.

c. Check the f2c output for correct typing of variables. F2c seems to require implicit Fortran typing for integers. For example, this doesn't work:

Subroutine mysub(order)

Integer* 4 order

But this does:

Subroutine mysub(norder)

2. Make sure that you have included yourcode.gxh in yourcode.gxc with the statement:

\#include "yourcode.gxh"

and that the library name in yourcode.gxh is correct and not longer than 11 characters.

3. Make sure the calls in $g x x \_y o u r c o d e . c$ are correct. See the following table. 


\begin{tabular}{|c|c|c|c|}
\hline \multirow[t]{2}{*}{ yourcode.f } & \multirow[t]{2}{*}{ yourcode.c } & \multicolumn{2}{|l|}{ gxx_yourcode.c } \\
\hline & & definition & call \\
\hline \multirow[t]{2}{*}{$\begin{array}{l}\left.\text { character }{ }^{\star}{ }^{\star}\right) \\
\text { infile }\end{array}$} & char *infile & $\begin{array}{l}\text { const char } \\
{ }_{\text {*pcInfile }}\end{array}$ & $\begin{array}{l}\text { const char } \\
{ }^{\star} \text { pcInfile }\end{array}$ \\
\hline & $\begin{array}{l}\text { ftnlen } \\
\text { infile_len }\end{array}$ & long *plInfile_len & *plInfile_len \\
\hline integer ${ }^{*}$ ntotal & integer *ntotal & long * pINotal & plNtotal \\
\hline Real*4 blat & real *blat & double *pdBlat & \&fBlat \\
\hline Real*8 ave & $\begin{array}{l}\text { doublereal } \\
\text { *ave }\end{array}$ & double *pdAve & pdAve \\
\hline
\end{tabular}

So the Fortran code (in yourcode.f):

subroutine mysub(infile, ntotal, blat, ave,ierr)

character $\left.{ }^{*}\right)$ infile

real* 8 ave

becomes the $C$ code (in yourcode.c):

int mysub_(char *infile, integer *ntotal, real *blat, doublereal *ave, integer *ierr, ftnlen infile_len)

which is referenced (in $g x x$ yourcode.c) via the wrapper function:

GX_WRAPPER_FUNC GX_LONG GX_WRAPPER_CALL

IUSGS_Mysub(void *pGeo,

const char *pcInfile, long *plinfile_len, 


$$
\begin{aligned}
& \text { double *pdblat, } \\
& \text { double *pdave) }
\end{aligned}
$$

\{

const char *modn = "IUSGS_Mysub";

float fBlat;

long $\mathrm{IErr}=0$;

// --- Load the global structure ---

InitGlobals(pGeo);

// --- Convert doubles to floats --

fBlat $=(\text { float })^{*}$ pdBlat:

mysub_((char *)pcInfile, pINtotal, \&fBlat, pdAve, \&IErr, *plInfile_len);

// --- Transfer returned values back to doubles --

${ }^{*}$ pdBlat = fBlat;

return 0;

\}

4. Use debugging options

A. Debug the gxc code using assert and abort:

Assert_SYS(i); // will display a mesage if $i$ is zero or FALSE. 

Abort_SYS("mesage"); // will stop execution and display the message.

B. Debug the Fortran code using wrapper functions. For example, you can check that parameters are being passed correctly to the DLL by:

1. placing the following calls near the top of your Fortran code:

Call RegisterErr_WF(1000,'yourcode')

Call SetErrParmS_WF $\left(1\right.$, infile, $\left.{ }^{\star}\right)$

Call SetErrParmI_WF(2,ntotal)

Call SetErrParmR_WF(3,blat)

2. adding an error file yourcode.err to the

$C: \backslash O A S I S m o n t a j \backslash g e r$ directory containing:

$\# 1000$

! infile $=\% 1$

! ntotal $=\% 2$, blat $=\% 3$

3. and registering the error file in the InitGlobals section of wfuncs.c:

IStrcpy_STR(pGeo, Globals.szErrFile,"yourcode.err", \&lSize); 


\section{FORTRAN CONVERSION SUMMARY}

yourcode.f

subroutine yourcode()
:
call RegisterErr_WF()
call
SetErrParmI_WF()
:
call SomeOther_WF()
:
return

Visual $C++6.0$

yourcode.dll

yourcode.g $\times h$

\#define

USGS_Yourcode()

yourcode.gxc

USGS_Yourcode()

yourcode.gr, yourcode.grh, yourcode.hlp

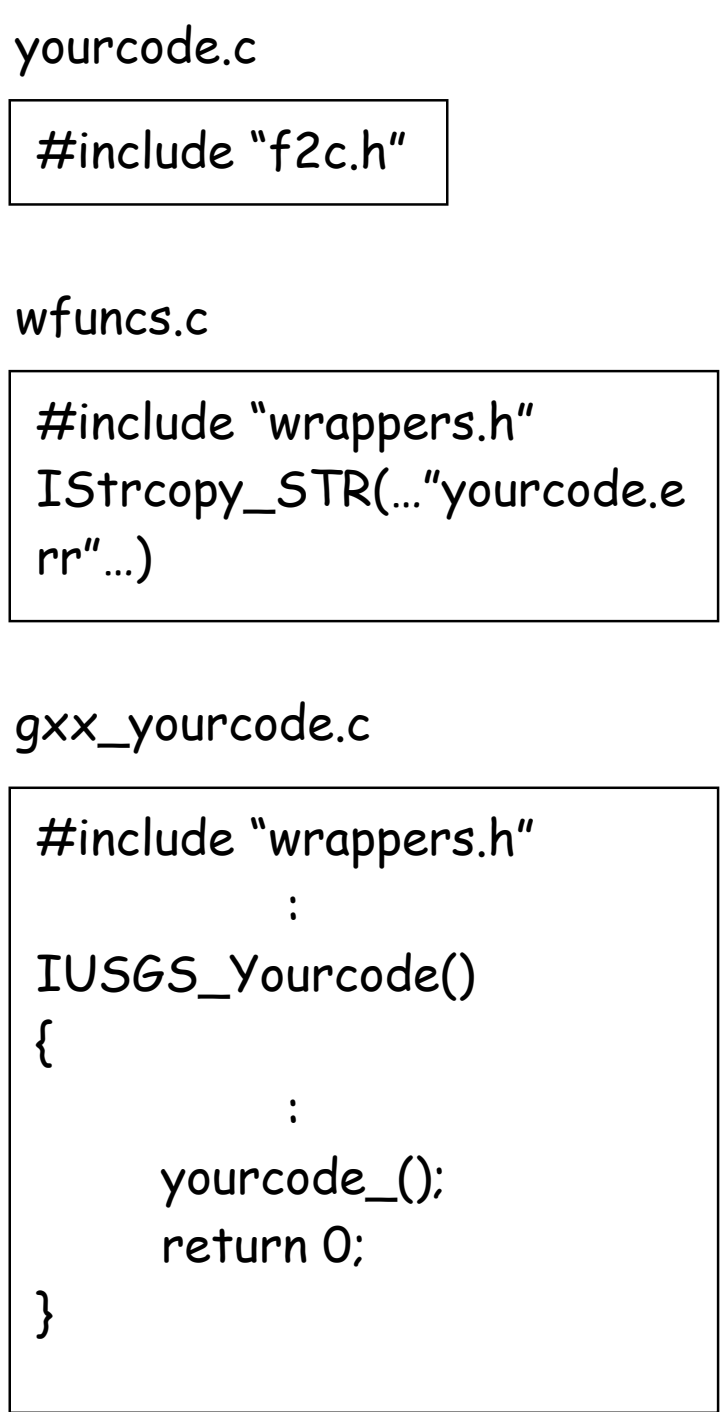
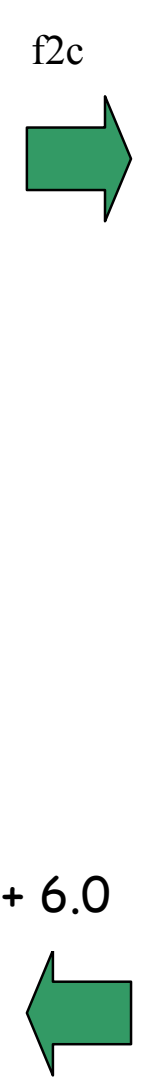